\title{
PI3K-alpha Inhibitor MEN1611
}

National Cancer Institute

\section{Source}

National Cancer Institute. PI3K-alpha Inhibitor MEN1611. NCI Thesaurus. Code C158603.

An orally bioavailable inhibitor of the class I phosphatidylinositol-4,5-bisphosphate 3kinase (PI3K) catalytic subunit alpha (PIK3CA), with potential antineoplastic activity. PI3K alpha inhibitor MEN1611 selectively binds to and inhibits PIK3CA and its mutated forms in the PI3K/Akt (protein kinase B)/mammalian target of rapamycin (mTOR) pathway. This results in both apoptosis and growth inhibition in PIK3CA-expressing tumor cells. By specifically targeting PIK3CA, this agent may be more efficacious and less toxic than panPI3K inhibitors. In addition, MEN1611 also targets mutated forms of PI3K gamma (PI3Kg). MEN1611 may stimulate the immune system to restore CD8+ T-cell activation and cytotoxicity. Dysregulation of the PI3K/Akt/mT OR pathway is often found in solid tumors and results in the promotion of tumor cell growth, survival, and resistance to chemo- and radio-therapy. PIK3CA, one of the most frequently mutated oncogenes, encodes the p110-alpha catalytic subunit of the class I PI3K. In most solid tumors, the activation of the PI3K pathway is induced by mutations of PIK3CA. 